\title{
Cesium alignment produced by pumping with unpolarized light ${ }^{\star}$
}

\author{
Yongqi Shi ${ }^{1,2,3, a}$ and Antoine Weis ${ }^{1}$ \\ 1 Physics Department, University of Fribourg, Chemin du Musée 3, 1700 Fribourg, Switzerland \\ 2 Institute of Electronics, Chinese Academy of Sciences, Beijing 100190, P.R. China \\ ${ }^{3}$ University of Chinese Academy of Sciences, Beijing 100049, P.R. China
}

\begin{abstract}
We demonstrate optical pumping on the four hyperfine components of the Cs $D_{1}$ transition by unpolarized (UPL) resonant laser light. The evidence is based on the reduction of the absorption coefficients $\kappa_{0}$ with increasing light power $P$ in an uncoated $\mathrm{Cs}$ vapor cell with isotropic spin relaxation. For comparison we perform the same quantitative $\kappa_{0}(P)$ measurements with linearly-polarized light (LPL) and circularly-polarized light (CPL). We find that our previously published algebraic expressions give an excellent description of all experimentally recorded induced transparency signals. Based on this we can make reliable absolute predictions for the power dependence of the spin orientation and alignment produced by pumping with LPL, CPL and UPL.
\end{abstract}

\section{Introduction}

Optical pumping is a method by which the (rotation and reflection) symmetry of resonant light is transferred to an atomic ensemble by a sequence of light absorptionemission cycles. In most cases, circularly-polarized light $(\mathrm{CPL})$ or linearly-polarized light (LPL) is deployed in optical pumping experiments. While CPL produces spin orientation and alignment along the light's propagation direction $\hat{k}$, pumping with LPL produces only spin alignment along the light's polarization direction.

As discussed, e.g., by Happer [1], pumping by unpolarized light (UPL) will also affect the symmetry of the atomic medium. The symmetry of UPL is determined by the symmetry of $\hat{k}$, so that optical pumping with UPL will produce alignment along $\hat{k}$. With a suitably chosen quantization axis, the spin polarization of a medium is fully described by the populations $p_{F, m}$ of its magnetic sublevels, as shown in the qualitative examples of Figure 1.

Pumping with LPL and UPL yields symmetric population distributions in the sense that $p_{F, m}=p_{F,-m}$, while pumping with CPL yields asymmetric populations $p_{F, m} \neq p_{F,-m}$. Because UPL (modeled herein as an incoherent 50/50 mixture of $\sigma_{+}$and $\sigma_{-}$CPL) affects $m$ and $-m$ states in the same manner, it will not optically pump atoms with an angular momentum of $1 / 2$.

In the early years of optical pumping, Franken and Colegrove have already demonstrated optically detected magnetic resonance in the metastable $2^{3} S_{1}$ state of ${ }^{4} \mathrm{He}$, based on the longitudinal alignment created in that state

${ }^{\star}$ Supplementary material

${ }^{a}$ e-mail: yongqi.shi@unifr.ch by pumping with UPL from a ${ }^{4} \mathrm{He}$ discharge lamp [2]. To our knowledge optical pumping of alkali atoms by UPL has remained largely unexplored. Because of the $J=1 / 2$ angular momentum of alkali ground states, optical pumping with spectrally-broad UPL from discharge lamps does not create any imbalance of the $m_{J}$ populations. However, laser experiments, in which the two $F_{ \pm}=I \pm 1 / 2$ hyperfine levels of the ground state are resolved, can be used for UPL pumping.

In this paper we demonstrate optical pumping in Cs atoms with UPL by studying the reduction of the resonant optical absorption coefficient with increasing light power. We compare the experimental results to those obtained with LPL and CPL. In a recent publication [3] we have studied optical pumping in a paraffin-coated Cs vapor cell, in which spin relaxation is dominated by anisotropic ( $F$ - and $m$-dependent) spin-exchange collisions. The measured quantities were the resonant absorption coefficients' dependence on laser power, $\kappa_{0}(P)$, for the four Cs $D_{1}$ hyperfine transitions. We found excellent quantitative agreement with numerical calculations taking the anisotropic spin relaxation into account. In that paper we have also shown that under the assumption of isotropic relaxation, one can derive algebraic expressions for the $\kappa_{0}(P)$ dependencies. Here we put these analytical results on the experimental test bench, by repeating the same experiments as described in reference [3] in an uncoated cell, which fulfills the condition of isotropic relaxation at room temperature.

\section{Theory}

The power $P$ of laser light, resonant with an $F_{g} \rightarrow$ $F_{e}$ transition, that has traversed an atomic medium of column length $L$ is given by $P=P_{\text {in }} \exp \left(-\kappa_{0} L\right)$ 

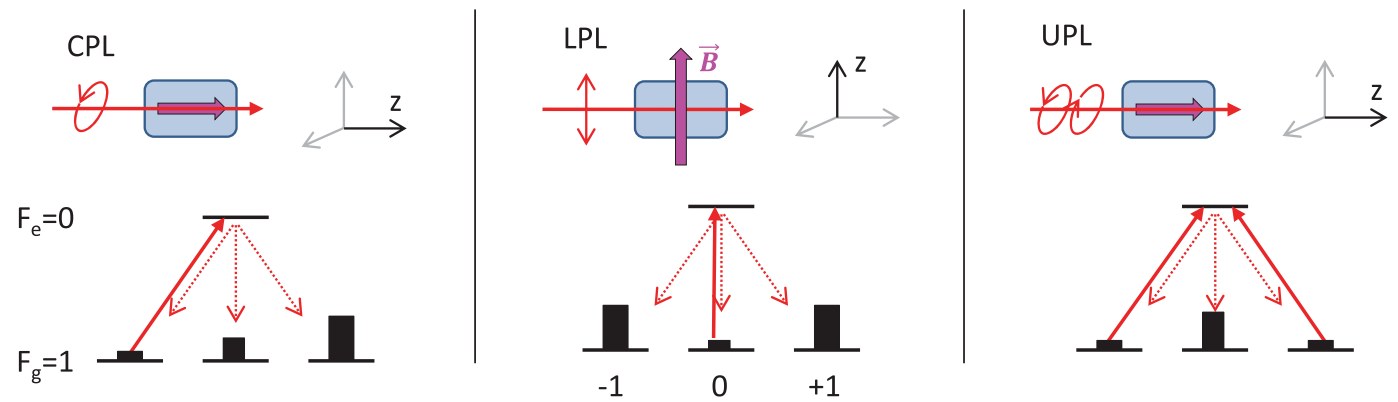

Fig. 1. Example of optical pumping on an $F_{g}=1 \rightarrow F_{e}=0$ transition with circularly polarized (left), linearly polarized (center) and unpolarized (right) light. A static magnetic holding field $\boldsymbol{B}$ stabilizes the produced spin polarization.

(Lambert-Beer law), where $P_{\text {in }}$ is the power entering the medium. Since optical pumping reshuffles (in general) sublevel populations to less absorbing states, $\kappa_{0}$ decreases (in general) with increasing laser power $P_{\text {in }}$. In reference [3] we have shown how the 16 steady-state sublevel populations of the Cs ground state $p_{F_{g}, m_{g}}$ produced by optical pumping with light of power $P_{\text {in }}$ and polarization "POL" tuned to the $F_{g} \rightarrow F_{e}$ hyperfine transition are obtained as steady-state solutions of the 16 coupled algebraic rate equations

$$
\sum_{j}\left(\mathbb{R}_{i j} x-\mathbb{D}_{i j} x-\delta_{i j}\right) p_{j}^{\mathrm{ss}}=p_{i}^{\mathrm{eq}}
$$

where $\mathbb{D}_{i j}\left(F_{g}, F_{e}, \mathrm{POL}\right)$ and $\mathbb{R}_{i j}\left(F_{g}, F_{e}, \mathrm{POL}\right)$ are the matrix representations of the depopulation and repopulation operators, respectively. $p_{j}^{\text {ss }}$ is the steady-state population of the $j$ th Zeeman sublevel. $p_{i}^{\text {eq }}$ are the thermal equilibrium populations when there is no pumping, considered to be $1 / 16$.

The dependence on the incident light power $P_{\text {in }}$ is expressed in terms of the dimensionless optical pumping saturation parameter

$$
x \equiv \frac{\Gamma_{\mathrm{abs}}}{\gamma_{1}} \equiv \frac{P_{\mathrm{in}}}{P_{\mathrm{sat}}}
$$

In equation (2), the optical pumping rate $\Gamma_{\mathrm{abs}}$ is given by

$$
\Gamma_{\mathrm{abs}}=\frac{4 \pi^{3 / 2} \alpha|\mathrm{r}|^{2}}{\hbar \Gamma_{\mathrm{D}}} \frac{P_{\mathrm{in}}}{S_{\mathrm{eff}}}
$$

where $\alpha$ is the fine structure constant, $S_{\text {eff }}=$ $\pi\left(D_{4 \sigma} / 2\right)^{2}$ the effective laser beam cross section, $|r| \equiv$ $\left|\left\langle 6 P_{1 / 2}\|r\| 6 S_{1 / 2}\right\rangle\right|$ the modulus of the reduced matrix element of the dipole operator, and

$$
\Gamma_{D}=\omega_{\mathrm{eg}} \sqrt{\frac{2 k_{B} T}{M_{\mathrm{Cs}} c^{2}}}
$$

the Doppler width, $M_{\mathrm{Cs}}$ being the Cs atom's mass, and $\omega_{\text {eg }}$ the optical transition frequency. Thus the saturation power in equation (2) is given by

$$
P_{\text {sat }}=\frac{\hbar \Gamma_{D} \gamma_{1} S_{\mathrm{eff}}}{4 \pi^{3 / 2} \alpha|r|^{2}}
$$

where $\gamma_{1}$ the longitudinal spin relaxation rate that is assumed to be isotropic, i.e., independent of $F_{g}$ and $m_{g}$.

The index $i$ in equation (1) labels the 16 sublevel populations $p_{F_{g}, m_{g}}$. The analytical solutions of the rate equations are of the analytical rational form

$$
p_{F_{g}, m_{g}}^{\stackrel{F_{g}}{\mathrm{POL}} F_{e}}(x)=\mathcal{A} \frac{\sum_{n} \mathcal{B}_{n} x^{n}}{\sum_{n} \mathcal{C}_{n} x^{n}} .
$$

Once the populations are known, the absorption coefficients can be calculated according to

$$
\kappa_{0}^{F_{g} \stackrel{\mathrm{POL}}{\longrightarrow} F_{e}}=\sum_{m_{g}=-F_{g}}^{+F_{g}} p_{F_{g}, m_{g}}^{\stackrel{\mathrm{POL}}{\longrightarrow} F_{e}} c_{F_{e}, m_{e} ; F_{g}, m_{g}}^{(\mathrm{POL})} \widetilde{C}_{\kappa},
$$

with

$$
\widetilde{C}_{\kappa}=4 \pi \alpha \sqrt{\frac{\pi M_{\mathrm{Cs}} c^{2}}{2 k_{B} T}} N_{\mathrm{Cs}}|r|^{2}
$$

where $N_{\text {Cs }}$ is the Cs number density. In equation (7), the $c$ coefficients are given by

$$
\begin{aligned}
& c_{F_{e}, m_{e} ; F_{g}, m_{g}}^{(\mathrm{UPL})}=\frac{\left(2 F_{g}+1\right)\left(2 F_{e}+1\right)}{2} \\
& \times\left[\left(\begin{array}{ccc}
F_{e} & 1 & F_{g} \\
-m_{g}+1 & -1 & m_{g}
\end{array}\right)^{2}+\left(\begin{array}{ccc}
F_{e} & 1 & F_{g} \\
-m_{g}-1 & +1 & m_{g}
\end{array}\right)^{2}\right] \\
& \times\left\{\begin{array}{l}
J_{e} J_{g} 1 \\
F_{g} F_{e} I
\end{array}\right\}^{2}, \\
& c_{F_{e}, m_{e} ; F_{g}, m_{g}}^{(\mathrm{LPL})}=\left(2 F_{g}+1\right)\left(2 F_{e}+1\right) \\
& \times\left(\begin{array}{ccc}
F_{e} & 1 & F_{g} \\
-m_{g} & 0 & m_{g}
\end{array}\right)^{2}\left\{\begin{array}{c}
J_{e} J_{g} 1 \\
F_{g} F_{e} I
\end{array}\right\}^{2},
\end{aligned}
$$



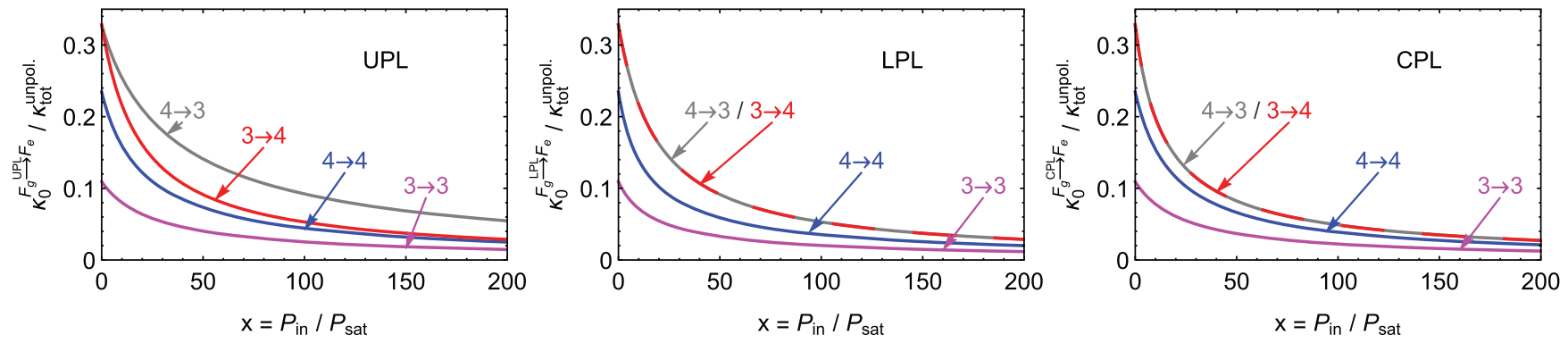

Fig. 2. Theoretical dependence of (normalized) absorption coefficient on dimensionless parameter $x=\Gamma_{\text {abs }} / \gamma_{1}=P_{\text {in }} / P_{\text {sat }}$ with unpolarized, linearly-polarized, and circularly-polarized light.

and

$$
\begin{aligned}
c_{F_{e}, m_{e} ; F_{g}, m_{g}}^{(\mathrm{CPL})}= & \left(2 F_{g}+1\right)\left(2 F_{e}+1\right) \\
& \times\left(\begin{array}{ccc}
F_{e} & 1 & F_{g} \\
-m_{g} \mp 1 & \pm 1 & m_{g}
\end{array}\right)^{2}\left\{\begin{array}{c}
J_{e} J_{g} 1 \\
F_{g} F_{e} I
\end{array}\right\}^{2}
\end{aligned}
$$

for the three polarization cases addressed in the paper.

Using the analytical expressions for the sublevel populations one thus gets analytical expressions for the (normalized) absorption coefficients of the form

$$
\frac{\kappa_{0}^{F_{g} \stackrel{\text { POL }}{\longrightarrow} F_{e}}(x)}{\kappa_{\text {tot }}^{\text {unpol. }}}=\mathcal{D} \frac{\sum_{n} \mathcal{E}_{n} x^{n}}{\sum_{n} \mathcal{F}_{n} x^{n}}
$$

where

$$
\kappa_{\text {tot }}^{\text {unpol. }}=\sum_{F_{g}=3,4} \sum_{F_{e}=3,4} \kappa_{0}^{F_{g} \stackrel{\text { POL }}{\longrightarrow} F_{e}}(x=0)=\frac{\widetilde{C}_{\kappa}}{6}
$$

is the total absorption coefficient when the medium is unpolarized, i.e., when all 16 sublevel populations are given by $p_{F_{g}, m_{g}}=1 / 16$.

We refer to the supplementary material for a listing of the numerical values of the coefficients $\mathcal{D}, \mathcal{E}_{n}$, and $\mathcal{F}_{n}$ - all functions of $F_{g}, F_{e}$, and POL - for all transitions and polarizations. Figure 2 shows graphical representations of the functions in equation (12). As addressed already in reference [3], pumping with CPL and LPL under conditions of isotropic relaxation yields identical power dependencies for the $3 \rightarrow 4$ and $4 \rightarrow 3$ transitions. This peculiar feature does not appear under pumping with UPL nor when the relaxation is anisotropic [3].

It is also worthwhile mentioning that all 12 curves are described by 2 (common) parameters only, viz., an overall common scale $\kappa_{\text {tot }}^{\text {unpol. }}$, determined by the single experimental parameter $N_{\mathrm{Cs}}(T)$ (Cs number density), and the saturation power $P_{\text {sat }}$ given by equation (5), determined by two experimental parameters, viz., the effective beam area $S_{\text {eff }}$ (cf. Sect. 4.2) and the vapor temperature $T$ entering via the Doppler width $\Gamma_{D}$ in equation (4). Knowing the path length $L$ of the laser beam in the vapor, one is left with two experimental parameters, $T$ and $S_{\text {eff }}$ that

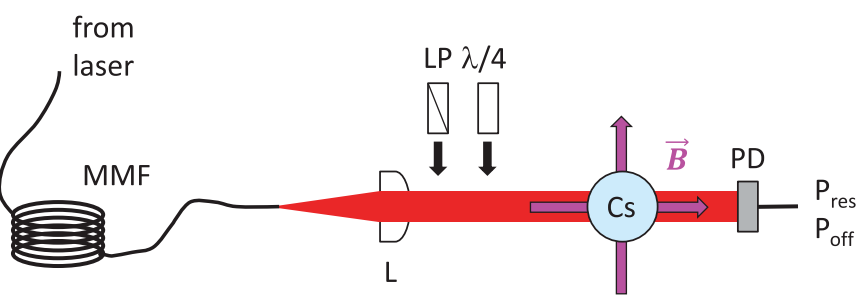

Fig. 3. Basic elements of the experimental set-up. MMF: multimode fiber, L: lens, LP: linear polarizer, $\lambda / 4$ : quarter-wave plate, Cs: cesium vapor cell, PD: photodiode. The multilayer $\mu$-metal shield is not shown. For the different experiments a static magnetic field $\boldsymbol{B}$ is applied along the directions shown in Figure 1.

fully determine all of the 12 (=3 polarizations $\times 4$ transitions) experimental $\kappa\left(P_{\text {in }}\right)$ dependencies on an absolute scale.

\section{Experiments}

The experimental setup (shown schematically in Fig. 3) is similar to the one described in reference [3], with the following modifications: The laser light is carried by a $20 \mathrm{~m}$-long multi-mode $(400 \mu \mathrm{m})$ fiber, wound into multiple 8-cm diameter loops which scramble the light polarization. The residual degree of polarization is $<4 \%$, as measured by a polarimeter (Thorlabs, model PAX5710IR1-T).

The atomic medium is Cs vapor at a saturated vapor pressure corresponding to room temperature that is contained in a spherical (28 mm inner diameter) Pyrex glass cell. The cell contains no buffer gas and has no wall coatings, so that spin relaxation is determined by the finite interaction time of the atoms with the laser beam (transittime broadening). The ensuing relaxation - at a rate of $\gamma_{1} /(2 \pi) \sim 16 \mathrm{kHz}$ - can be considered as isotropic, in contrast to the paraffin-coated cell used in our previous experiment [3], in which relaxation - at a rate $\gamma_{1} /(2 \pi)$ of a few $\mathrm{Hz}$ - was dominated by anisotropic spin-exchange collisions.

The light from the multi-mode fiber is collimated into a beam with a $D_{4 \sigma}$ diameter [4] of $\sim 1.5 \mathrm{~mm}$ prior to entering the cell. For experiments with LPL and CPL, a polarizer or a polarizer $-\lambda / 4$ combination is inserted between the lens and the vapor cell. 

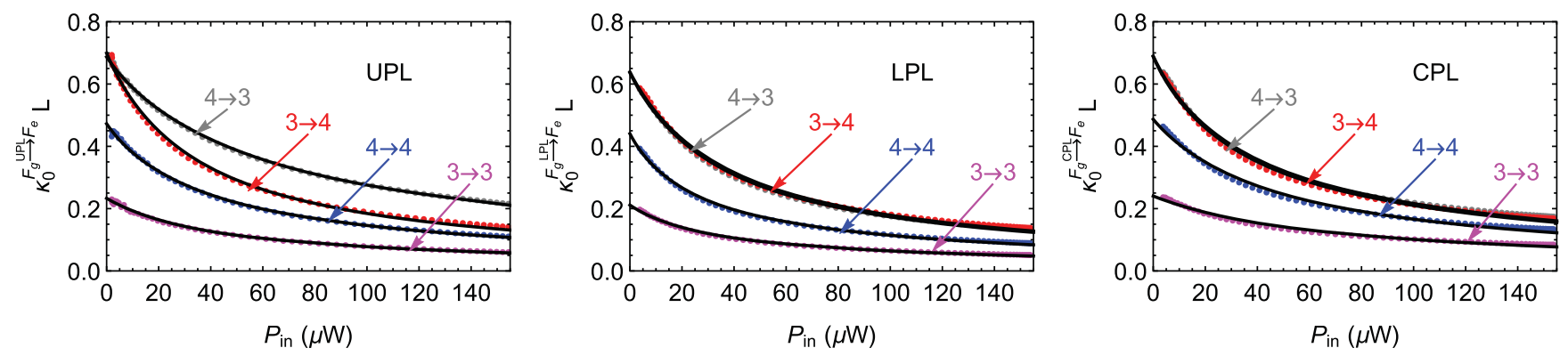

Fig. 4. Experimental data (colored dots) and fitted dependencies (solid lines) according to equation (15).

A magnetic holding field $\boldsymbol{B}$ of $40 \mu \mathrm{T}$ (corresponding Larmor frequency $\omega_{L} \sim 9 \gamma_{1}$ ) is applied along $\boldsymbol{k}$ in the experiments with CPL and UPL, while the holding field is oriented along the light polarization in the LPL experiment (cf. Fig. 1).

In order to record the $\kappa_{0}\left(P_{\text {in }}\right)$ dependencies of the four hyperfine components we used the method described in reference [3]. The laser frequency is locked to the center of a specific $F_{g} \stackrel{\text { POL }}{\longrightarrow} F_{e}$ transition, and the laser power $P_{\text {in }}$ is scanned from 1 to $\sim 150 \mu \mathrm{W}$ using the arbitrary light power controller described in reference [5]. The maximum value of $P_{\text {in }}$ was chosen such as to illustrate well how the $3 \rightarrow 4$ curve splits off from the $4 \rightarrow 3$ one, and approaches the $4 \rightarrow 4$ curve for UPL pumping. Recording the transmitted power $P_{\text {res }}$, these scans yield recordings $P_{\text {res }}\left(P_{\text {in }}\right)$. We also perform a similar scan with the laser frequency set to a far off-resonance frequency, recording $P_{\text {off }}\left(P_{\text {in }}\right)$ dependencies. After calibrating $P_{\text {off }}$ against $P_{\text {in }}$ with a calibrated power meter, this procedure allows us to infer $\kappa_{0} L$ from the Lambert-Beer law

$$
\kappa_{0} L\left(P_{\text {in }}\right)=-\ln \frac{P_{\text {res }}\left(P_{\text {in }}\right)}{P_{\text {off }}\left(P_{\text {in }}\right)} .
$$

Following this procedure we have recorded a total of 12 (4 transitions, 3 light polarizations) experimental optical thickness values $\kappa_{0}^{F_{g} \stackrel{\text { POL }}{\longrightarrow} F_{e}}\left(P_{\text {in }}\right) L$.

\section{Results}

\subsection{Fitting procedure}

Figure 4 shows the experimental $\kappa_{0}^{F_{g} \stackrel{\text { POL }}{\longrightarrow} F_{e}}\left(P_{\text {in }}\right) L$ dependencies values. We have fitted each of the 12 curves by a function

$$
f\left(P_{\mathrm{in}}\right)=\widetilde{A}^{F_{g}} \stackrel{\text { POL }}{\longrightarrow} F_{e} \frac{\kappa_{0}^{F_{g}} \stackrel{\text { POL }}{\longrightarrow} F_{e}\left(P_{\mathrm{in}} / \widetilde{P}_{\mathrm{sat}}^{F_{g}} \stackrel{\text { POL }}{\longrightarrow} F_{e}\right)}{\kappa_{\text {tot }}^{\text {unpol. }}},
$$

where $\kappa_{0}^{F_{g} \stackrel{\text { POL }}{\longrightarrow} F_{e}}\left(x=P_{\text {in }} / \widetilde{P}_{\text {sat }}^{F_{g} \stackrel{\text { POL }}{\longrightarrow} F_{e}}\right) / \kappa_{\text {tot }}^{\text {unpol. }}$ are the theoretical expressions given in algebraic form by equation (12). Each fit is thus determined by the two fit

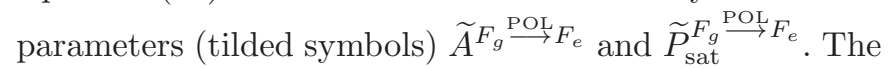

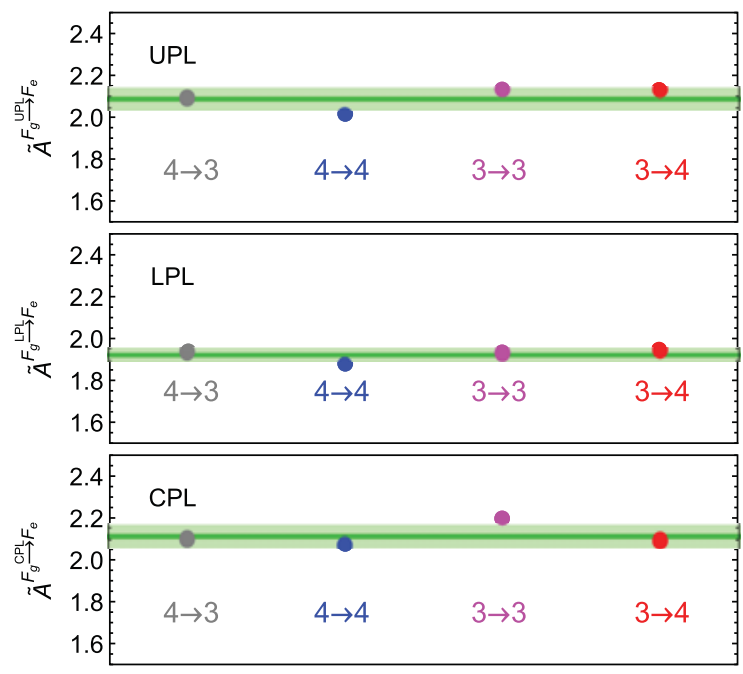

Fig. 5. Fitted amplitude parameters $\widetilde{A}^{F_{g}} \stackrel{\text { POL }}{\longrightarrow} F_{e}$ and their weighted averages (horizontal bands).

fitted curves give an excellent match with the experimental data, as shown in Figure 4.

Figures 5 and 6 show the fitted parameter values, the average values of which (for each polarization) are reproduced in Table 1.

The errors on $\widetilde{A}^{\mathrm{POL}}$ and $\widetilde{P}_{\text {sat }}^{\mathrm{POL}}$ in the table are parameters uncertainties produced by the fitting software, while the uncertainties on $T$ and $D_{4 \sigma}$ were obtained by error propagation from the latter, respectively.

\subsection{Interpreting the fit parameters}

The theoretical expression for the amplitude fit parameter is given by

$$
A^{F_{g} \stackrel{\text { POL }}{\longrightarrow} F_{e}}=\kappa_{\text {tot }}^{\text {unpol. }} L=\frac{2 \pi \alpha}{3}|r|^{2} \sqrt{\frac{\pi M_{\mathrm{Cs}} c^{2}}{2 k_{B} T}} N_{\mathrm{Cs}}(T) L .
$$

Using $L=28(1) \mathrm{mm}, \quad|r|=4.4978 a_{0} \quad\left(a_{0}\right.$ being the Bohr radius) [6], and the vapor pressure formula from reference [7], one can extract the cell temperature $T$ from the fitted values of $\tilde{A}^{F_{g}} \stackrel{\text { POL }}{\longrightarrow} F_{e}$. The results, shown in Table 1, are reasonable since they are compatible with the day-to-day temperature variations in the lab. 


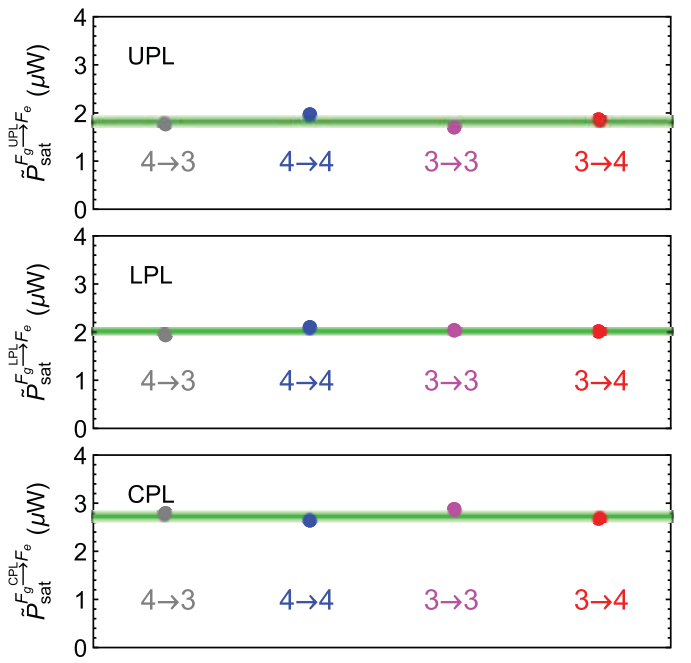

Fig. 6. Fitted saturation power parameters $\widetilde{P}_{\text {sat }}^{F_{g} \stackrel{\text { POL }}{\longrightarrow} F_{e}}$ and their weighted averages (horizontal bands).

Table 1. Weighted averages of the fit parameters $\widetilde{A}^{F_{g}} \stackrel{\text { POL }}{\longrightarrow} F_{e}$ and $\widetilde{P}_{\text {sat }}^{F_{g}} \stackrel{\text { POL }}{\longrightarrow} F_{e}$ for each light polarization, and derived vapor cell temperatures $T$ and $D_{4 \sigma}$ laser beam diameters.

\begin{tabular}{lllll}
\hline POL & $\widetilde{A}^{\mathrm{POL}}$ & $T\left({ }^{\circ} \mathrm{C}\right)$ & $\widetilde{P}_{\mathrm{sat}}^{\mathrm{POL}}(\mu \mathrm{W})$ & $D_{4 \sigma}(\mathrm{mm})$ \\
\hline $\mathrm{UPL}$ & $2.09(3)$ & $21.2(1)$ & $1.8(1)$ & $1.1(1)$ \\
$\mathrm{LPL}$ & $1.92(2)$ & $20.4(1)$ & $2.0(1)$ & $1.2(1)$ \\
$\mathrm{CPL}$ & $2.11(3)$ & $21.3(1)$ & $2.7(1)$ & $1.6(1)$ \\
\hline
\end{tabular}

The theoretical expression for the saturation power is given by

$$
P_{\mathrm{sat}}=\frac{\hbar \Gamma_{D} \gamma_{1} S_{\mathrm{eff}}}{4 \pi^{3 / 2} \alpha|r|^{2}}=\frac{1}{8 \sqrt{2 \pi}} \frac{\hbar \omega_{\mathrm{eg}} k_{B} T}{\alpha|r|^{2} M_{\mathrm{Cs}} c} D_{4 \sigma},
$$

where $\omega_{\text {eg }}$ is the optical transition frequency, and where the effective laser beam cross section is $S_{\text {eff }}=\pi\left(D_{4 \sigma} / 2\right)^{2}$. We assume that the spin relaxation rate $\gamma_{1}$ is the inverse of the atoms' time of flight through the laser beam, viz.,

$$
\gamma_{1}=\frac{v_{r}}{D_{4 \sigma}} \text { with the radial velocity } v_{r}=\sqrt{\frac{k_{B} T}{M_{\mathrm{Cs}}}}
$$

yielding an average value $\left\langle\gamma_{1}\right\rangle=(2 \pi) \times 16(2) \mathrm{kHz}$ using the average value of $T$ inferred from the amplitude fit parameters. Equating the fitted $\widetilde{P}_{\text {sat }}$ values with the last expression in equation (16) allows us then to infer the $4 \sigma$ beam diameters. The values (listed in Tab. 1) agree reasonably well with the experimental determination $D_{4 \sigma}=$ $1.5(3) \mathrm{mm}$ by a CCD camera placed before and after the cell, considering that the profile of the laser beam delivered by the multimode fiber is highly non-Gaussian.

\section{Discussion}

The induced transparency in the $\kappa_{0}(x)$ dependencies is due to the build-up of atomic spin polarization. As
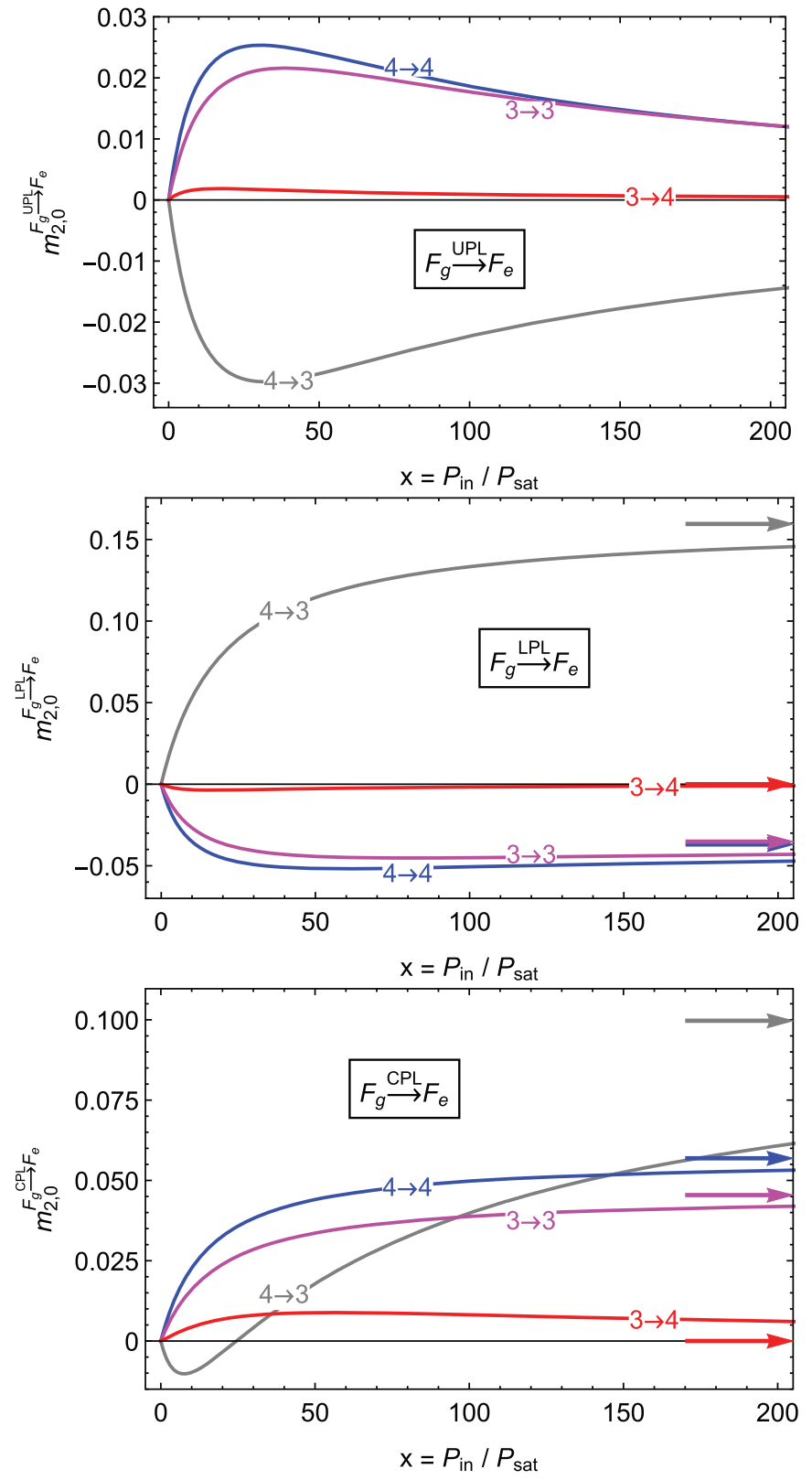

Fig. 7. Dependence of the alignment $m_{2,0}$ created in the state $F_{g}$ when pumped with UPL, LPL, and CPL (from top to bottom) on the $F_{g} \rightarrow F_{e}$ hyperfine transition. The arrows on the right indicate the asymptotic values reached for $x \rightarrow \infty$. In the case of UPL pumping all alignments vanish in that limit.

discussed in Appendix A, spin polarization is best characterized by the longitudinal atomic multipole moments $m_{1,0}$ (orientation) and $m_{2,0}$ (alignment). Both quantities can be expressed - similarly to the expressions for the sublevel populations - as rational functions of $x=P_{\text {in }} / P_{\text {sat }}$ that are given in Appendix A. Figure 7 shows the $m_{2,0}(x)$ dependencies for the three polarization cases addressed in the paper. The curves show the following features:

- The alignment in the $F_{g}=4$ state produced by pumping with UPL reaches a maximum value $\left|m_{2,0}(x)\right|$ of $\approx 0.03$ (when pumping either on the 
$4 \rightarrow 3$ or the $4 \rightarrow 4$ transition) and vanishes as $x \rightarrow \infty$.

- The alignment in the $F_{g}=3$ state produced by pumping with UPL reaches a maximum value $m_{2,0}(x)$ of $\approx 0.02$, when pumping on the $3 \rightarrow 3$ transition, while the maximum alignment achieved on the $4 \rightarrow 4$ transition is $\ll 0.01$. Here too, the alignment vanishes as $x \rightarrow \infty$.

- In case one wishes to create alignment in the $F_{g}=4$ state, pumping on the $4 \rightarrow 3$ transition with LPL is by far the most efficient, reaching an asymptotic value of 0.16 for $x \rightarrow \infty$, half of which (i.e. 0.08 ) is reached for a power $P_{\text {in }}$ of $\sim 20 P_{\text {sat }}$.

- Quite surprisingly, pumping with CPL allows to achieve a comparatively large asymptotic alignment of 0.1 when pumping on the $4 \rightarrow 3$ transition.

- Pumping with CPL on the $4 \rightarrow 4$ and $3 \rightarrow 3$ transitions produces alignment that is still approximately twice as large as pumping with UPL.

- Finally, we note a peculiar feature that occurs with CPL pumping. The produced alignment of the $F_{g}=$ 4 state, when pumped on the $4 \rightarrow 3$ transition vanishes for $P_{\text {in }}=24.5 P_{\text {sat }}$. This fact is of particular interest in the following context: In previous precision optical pumping experiments $[8,9]$ with CPL, we have tentatively assigned slight deviations of experimental signals from model dependencies to the omission of alignment contributions in the modeling. Studying the laser dependence of such irregularities in the region of vanishing alignment may prove useful to shine more light on these observations.

\section{Summary}

We have measured the reduction of the resonant optical absorption coefficient $\kappa_{0}$ of atomic cesium vapor induced by resonant optical pumping on the four hyperfine components of the $D_{1}$ transition with unpolarized laser light. By this we have demonstrated that optical pumping with resonant UPL can spin-polarize the Cs ground state. Because of the pumping light's symmetry, the symmetry of the created spin polarization is a rank $k=2$ tensor, i.e., an atomic alignment. We have made comparative measurements of the dependence of $\kappa_{0}$ on the incident light power $P_{\text {in }}$ for UPL, LPL and CPL polarizations.

We have developed model calculation based on algebraic solutions of optical pumping rate equations which yield algebraic expressions of the $\kappa_{0}\left(P_{\text {in }}\right)$ dependencies. The experimental recordings are very well fitted by the model expressions using two fit parameters (an overall scale factor and the optical saturation parameter), whose fitted values are well explained by two experimental parameters, viz., the vapor temperature and the laser beam diameter.

We used the thus verified algebraic expressions for the sublevel populations to produce algebraic expressions for the power dependence of the spin orientation and alignment in Cs vapor under pumping with UPL, LPL, and CPL. These results pave the way for a future fully algebraic modeling of a variety of spin physics studies, such as level crossing (Hanle effect), double resonance, and pump-probe experiments, on an absolute scale using the three step model described, e.g., in reference [10].

The authors acknowledge useful discussions and help with many practical issues by Victor Lebedev, Zoran Grujić, Theo Scholtes and Vladimir Dolgovskiy.

\section{Author contribution statement}

All the authors were involved in the preparation of the manuscript. All the authors have read and approved the final manuscript.

\section{Appendix A: Spin orientation and alignment}

Spin-polarized atomic media are conveniently described by their density matrix, which can be decomposed into multipole moments $m_{k, q}$ [11]. The absorption of an unpolarized medium is fully determined by the $k=0$ moment, $m_{0,0}$, that is proportional to the state population. It is well known [1] that the absorption coefficient $\kappa_{0}$ for light that drives an electric dipole transition in a spin-polarized medium depends only on the multipole moments of rank $k=1$ (vector polarization, spin orientation) and rank $k=2$ (tensor polarization, alignment). With a suitably chosen quantization axis (as shown for the cases discussed here in Fig. 1) for which the density matrix $\rho$ is diagonal, $\rho$ depends only on longitudinal $(q=0)$ moments $m_{k, 0}$ that are fully described by the magnetic sublevel populations $p_{F_{g}, m_{g}}$.

The absorption coefficient of CPL depends only on the longitudinal orientation $m_{1,0} \propto\left\langle\mathbb{F}_{z}\right\rangle$ and on the longitudinal alignment $m_{2,0} \propto\left\langle 3 \mathbb{F}_{z}^{2}-\mathbb{F}^{2}\right\rangle$, where $\mathbb{F}$ and $\mathbb{F}_{z}$ are the total angular momentum operator and its $z$-component, respectively. These moments can be expressed in terms of the sublevel populations as

$$
m_{1,0}(x)=\sqrt{\frac{3}{F_{g}\left(F_{g}+1\right)\left(2 F_{g}+1\right)}} \sum_{m_{g}=-F_{g}}^{F_{g}} m_{g} p_{F_{g}, m_{g}}(x),
$$

and

$$
\begin{aligned}
m_{2,0}(x)= & \sqrt{\frac{5}{\left(2 F_{g}-1\right) F_{g}\left(F_{g}+1\right)\left(2 F_{g}+1\right)\left(2 F_{g}+3\right)}} \\
& \times \sum_{m_{g}=-F_{g}}^{F_{g}}\left[3 m_{g}^{2}-F_{g}\left(F_{g}+1\right)\right] p_{F_{g}, m_{g}}(x) .
\end{aligned}
$$

Using the algebraic expressions equation (12) for the state populations, one can thus derive algebraic expressions for the power dependence of the relevant multipole moments of the form

$$
m_{1,0}(x)=\mathcal{H} \frac{\sum_{n} \mathcal{I}_{n} x^{n}}{\sum_{n} \mathcal{J}_{n} x^{n}} \text { and } m_{2,0}(x)=\mathcal{K} \frac{\sum_{n} \mathcal{L}_{n} x^{n}}{\sum_{n} \mathcal{M}_{n} x^{n}} .
$$




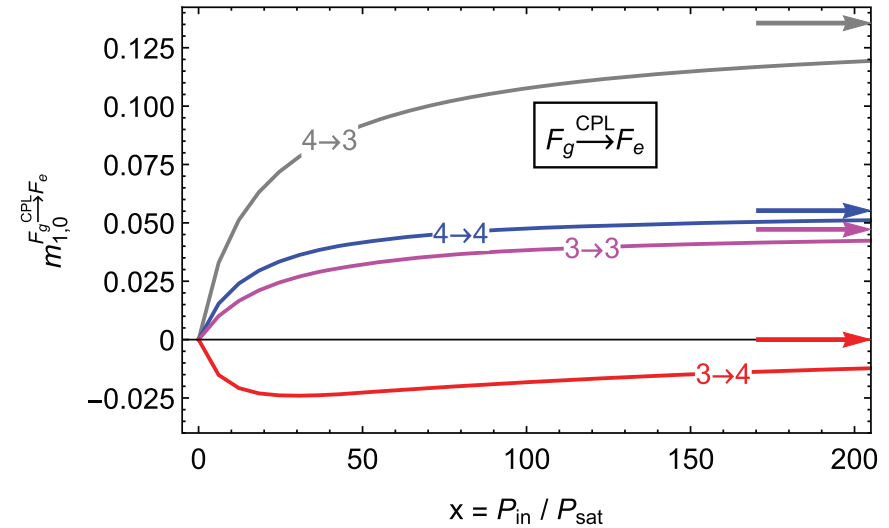

Fig. A.1. Dependence of the orientation $m_{1,0}$ created in the state $F_{g}$ when pumped with $\mathrm{CPL}$ on the $F_{g} \rightarrow F_{e}$ hyperfine transition. The arrows on the right indicate the asymptotic values reached for $x \rightarrow \infty$.

Tables with the numerical values of the parameters $\mathcal{H}$, $\mathcal{I}_{n}, \mathcal{J}_{n}, \mathcal{K}, \mathcal{L}_{n}$, and $\mathcal{M}_{n}$ are given as supplementary material. Graphical representations of the power dependence of $m_{2,0}$ for UPL, LPL and CPL pumping are shown in the main text (Fig. 7). For completeness, we show in Figure A.1 also the theoretical dependence of the spin orientation $m_{1,0}$ on laser power.

\section{References}

1. W. Happer, Rev. Mod. Phys. 44, 169 (1972)

2. P.A. Franken, F.D. Colegrove, Phys. Rev. Lett. 1, 316 (1958)

3. Y. Shi, T. Scholtes, Z.D. Grujić, V. Lebedev, V. Dolgovskiy, A. Weis, Phys. Rev. A 97, 013419 (2018)

4. A.E. Siegman, OSA trends in optics and photonics, in DPSS (diode pumped solid state) lasers: applications and issues, edited by M. Dowley (Optical Society of America, Washington, DC, 1998), Vol. 17, p. MQ1

5. Z.D. Grujić, J. Piller, A. Weis, Rev. Sci. Instrum. 89, $025114(2018)$

6. R.J. Rafac, C.E. Tanner, A.E. Livingston, K.W. Kukla, H.G. Berry, C.A. Kurtz, Phys. Rev. A 50, R1976 (1994)

7. M. Rozwadowski, E. Lipworth, J. Chem. Phys. 43, 2347 (1965)

8. Z.D. Grujić, A. Weis, Phys. Rev. A 88, 012508 (2013)

9. S. Colombo, V. Dolgovskiy, T. Scholtes, Z.D. Grujić, V. Lebedev, A. Weis, Exploring the world with the laser, edited by D. Meschede, T. Udem, T. Esslinger (Springer, Cham, 2018), p. 309

10. D. Budker, W. Gawlik, D.F. Kimball, S.M. Rochester, V.V. Yashchuk, A. Weis, Rev. Mod. Phys. 74, 1153 (2002)

11. K. Blum, Density matrix theory and applications, 3rd edn. (Springer-Verlag, Berlin, Heidelberg, 2012) 